\title{
Symmetry Broken in Low Dimensional N-body Chaos
}

\author{
David C. $\mathrm{Ni}^{1}$ and Chou Hsin Chin ${ }^{2}$
}

\author{
${ }^{1}$ Direxion Technology, 9F, 177-1 Ho-Ping East Road Section 1, \\ Taipei, Taiwan, R.O.C. \\ Email: davideni@yahoo.com \\ ${ }^{2}$ Department of Electro-Physics, National Chiao Tung University, \\ Hsin Chu, Taiwan, R.O.C. \\ Email: jchchin@,cc.nctu.edu.tw
}

\begin{abstract}
In this paper, we explore domains in conjunction with the defined parametric space of function $f=\mathrm{h}(\mathrm{z}) \prod_{\mathrm{i}}\left\{\exp \left(\mathrm{g}_{\mathrm{i}}(\mathrm{z})\right)\left[\left(\mathrm{a}_{\mathrm{i}}-\mathrm{z}\right) /\left(1-\overline{\mathrm{a}}_{\mathrm{i}} \mathrm{z}\right)\right]\right\}$, which represents a $\mathrm{N}$-body system in complex form. Interested observations on the computed results reveal limited levels of Herman Ring Fractals, Symmetry Broken, and transition to chaos based on phase parameter $\exp \left(\mathrm{g}_{\mathrm{i}}(\mathrm{z})\right)$ and $\mathrm{a}_{\mathrm{i}}$. Broken.

Keywords: N-body Chaos, Herman Ring Fractals, Meromophic, Symmetry
\end{abstract}

\section{Introduction}

Herman Rings represent a class of fractals with hierarchical structure in meromorphic dynamical systems. Due to the richness of mathematical elaboration and computing sophistication, we have observed limited efforts on simple rational functions $[1,2,3$, and 4$]$. In the previous efforts, we explored complex function: $\mathrm{h}(\mathrm{z}) \prod_{4}\left\{\exp \left(\mathrm{g}_{\mathrm{i}}(\mathrm{z})\right)\left[\left(\mathrm{a}_{\mathrm{i}}-\mathrm{z}\right) /\left(1-\overline{\mathrm{a}}_{\mathrm{i}} \mathrm{z}\right)\right]\right\}$ and revealed some interested characteristics of this function [5]. We extended our observations to the applications in antenna areas $[6,7]$ and elaborated the mathematical foundations [8].

We have then classified the function $\mathrm{f}=\mathrm{h}(\mathrm{z}) \prod_{\mathrm{i}}\left\{\exp \left(\mathrm{g}_{\mathrm{i}}(\mathrm{z})\right)\left[\left(\mathrm{a}_{\mathrm{i}}-\mathrm{z}\right) /\left(1-\overline{\mathrm{a}}_{\mathrm{i}} \mathrm{z}\right)\right]\right\}$ based on the special characteristics of this function [9]:

(a) There are at most five levels of partial and localized Herman Ring domains at different complex number (z) scales.

(b) For the orders, which are equal and higher than fourth orders, there exist five and only five level domains.

(c) The normalized phase and energy level parameters can induce symmetry broken.

In this paper, we examine in details about the symmetry broken and transition from stable fractal domains to chaotic domains based on two parameters: $\exp \left(\mathrm{g}_{\mathrm{i}}(\mathrm{z})\right)$ and $\mathrm{a}_{\mathrm{i}}$ in the paramtric space as listed in item (c) above. 


\section{Funcetion and Parametric Space}

\subsection{Function}

The function set, $\mathrm{f}=\mathrm{z}^{\mathrm{q}} \Pi C_{\mathrm{i}}$, with $\mathrm{h}(\mathrm{z})=\mathrm{z}^{\mathrm{q}}$, may have the following forms:

$$
\begin{aligned}
& \mathrm{f}=\mathrm{z}^{\mathrm{q}} \mathrm{C}_{1} \mathrm{C}_{2}, \\
& \mathrm{f}=\mathrm{z}^{\mathrm{q}} \mathrm{C}_{1} \mathrm{C}_{2} \mathrm{C}_{3},
\end{aligned}
$$

Where $\mathrm{z}$ is a complex variable, $\mathrm{q}$ is an integer and $\mathrm{C}_{\mathrm{i}}$ has following form:

$$
\mathrm{C}_{\mathrm{i}}=\exp \left(\mathrm{g}_{\mathrm{i}}(\mathrm{z})\right)\left[\left(\mathrm{a}_{\mathrm{i}}-\mathrm{z}\right) /\left(1-\overline{\mathrm{a}}_{\mathrm{i}} \mathrm{z}\right)\right]
$$

Here $\bar{a}_{i}$ is the complex conjugate of complex number $a_{i}$ and $z$ is also a complex number. We propose this form based on the following form known in the theory of special relativity by A. Einstein:

$$
\mathrm{u}_{\mathrm{x}}{ }^{\prime}=\left(\mathrm{u}_{\mathrm{x}}-\mathrm{v}\right) /\left(1-\mathrm{u}_{\mathrm{x}} \mathrm{v} / \mathrm{c}^{2}\right)
$$

The $\mathrm{z}^{\mathrm{q}}$ term in equation (1) has implication of time and interaction of $\mathrm{C}_{\mathrm{i}}$. We set $q=-1$ given the bondary of involved domains will become zero at infinity and the potential energy of interaction is propotional to reverse of distance. The term

$$
\exp \left(g_{i}(z)\right)
$$

In equation (2) represents the phase, where $\mathrm{g}_{\mathrm{i}}(\mathrm{z})$ is a complex function. A given domain can be a domain of complex numbers, $z=x+y i$, with $\left(x^{2}+y^{2}\right)^{1 / 2} \leqq R$. Here, $\mathrm{R}$ is a real number. We also use a simpler domain, $\left(\mathrm{x}^{2}+\mathrm{y}^{2}\right)^{1 / 2}=\mathrm{R}$, to observe directly a mapping or transformation from original domain to the set of $x+y i$ after functional iteration. There are two sets of domains in conjunction with the function: the converged set remained from original domains after several orders of functional iteration, and the mapped domains of original domains after several orders of functional iterations. The function $f$ will go through iteration as:

$$
f^{\mathrm{n}}(\mathrm{z})=f \circ f^{\mathrm{n}-1}
$$

Here, $\mathrm{n}$ is a positive integer indicating the order of functional iterations.

\subsection{Parametric Space}

To obtain the remained domains and mapped domains, we have to determine the criteria of convergence, divergence, and oscillation of functional values after several iterations. We adopted functional values of several consecutive iterations for this purpose. We define the parametric space or normalized space including the following parameters: 
(1) $\mathrm{z}$ - original or mapped domains

(2) a-normalized energy level of interaction

(3) $\exp \left(\mathrm{g}_{\mathrm{i}}(\mathrm{z})\right)$ - normalized phase factor

(4) $q$ - normalized distance factor of interaction

(5) Iteration

These parameters can transform stable domains into chaotic domains.

\section{Original and Mapped Domains}

Figure 1 (a) through (e) show the $\mathrm{Z}^{-1} \mathrm{C}_{1} \mathrm{C}_{2} \mathrm{C}_{3} \mathrm{C}_{4}$ original domains:

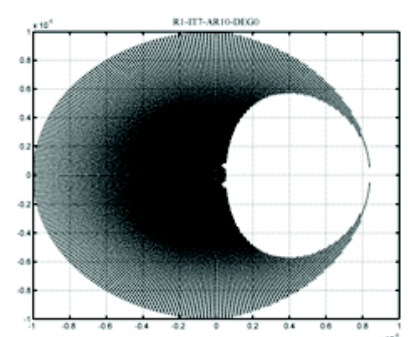

Figure 1 (a) $\mathrm{R} \sim 10^{-6}$

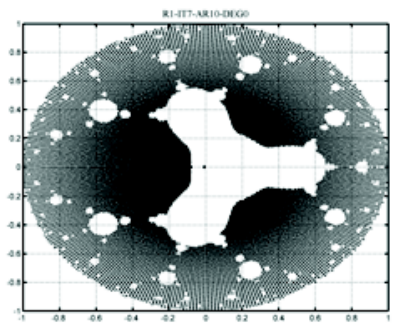

Figure 1(c) $\mathrm{R}=1$

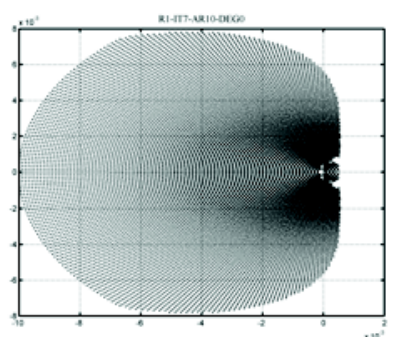

Figure $1(\mathrm{~b}) \mathrm{R} \sim 10^{-2}$

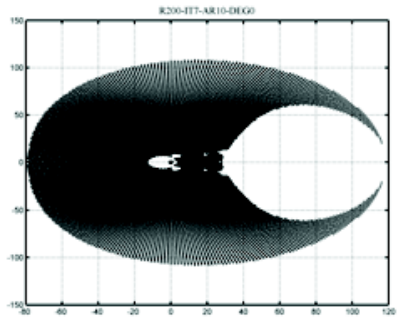

Figure $1(\mathrm{~d}) \mathrm{R} \sim 10^{2}$

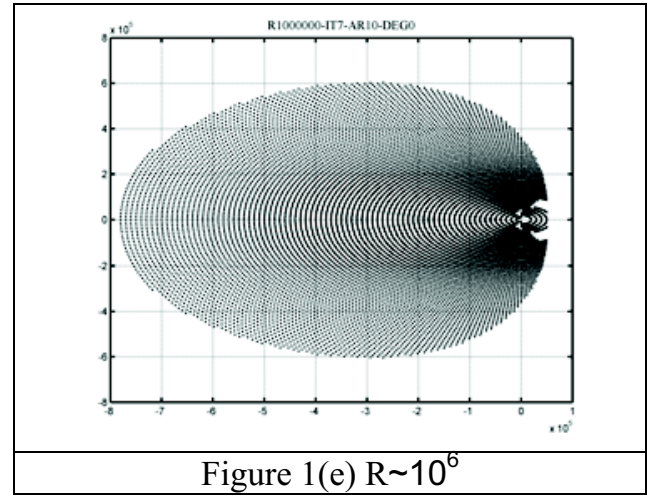


Figure 1(a), (b), (c), (d) and (e) show a original domain $\mathrm{z}=\mathrm{x}+\mathrm{yi}$, with $\left(\mathrm{x}^{2}\right.$ $\left.+\mathrm{y}^{2}\right)^{1 / 2} \leqq \mathrm{R}$, forming a set of 5-level of co-center partial Herman-Ring fractal domains at diiferent scales from a set of parameters in parametric space. Figure 1(a) shows a partial Herman-Ring domain at scale of $\mathrm{R} \sim 10^{-6}$. Figure 1 (b) shows domain a partial Herman-Ring domain at scale of $R \sim 10^{-2}$. Figure 1 (c) shows a partial Herman-Ring domain at scale of $\mathrm{R}=1$, the unit circle. The domain in Figure 1(b) can be seen locating around $\mathrm{z}=0$ point at Figure 1(c). Figure 1 (d) shows a partial Herman-Ring domain at scale of $\mathrm{R} \sim 10^{2}$. Figure 1(c) can be seen locating around $z=0$ point at Figure 1(d). Figure 1(e) shows a partial Herman-Ring domain at scale of $R \sim 10^{6}$. Figure 1 (d) is locating around $\mathrm{Z}=0$ point at Figure 1 (e). The partial Herman-Ring domain at scale of $\mathrm{R} \sim 10^{-6}$ is topologically similar to that at $\mathrm{R} \sim 10^{2}$ and the domain at scale of $\mathrm{R} \sim 10^{-2}$ is topologically similar to that at $\mathrm{R} \sim 10^{6}$. We call this as skip-level symmetry. For higher functional order than the fourth order (i.e., $f=Z^{-1} C_{1} C_{2} C_{3} C_{4}$ ), the number of levels keeps the same as $Z^{-1} C_{1} C_{2} C_{3} C_{4}$, namely, only 5 levels are found at $11^{\text {th }}$ order or $20^{\text {th }}$ order as examples. The skip-level symmetry is also observed in the higher-ordered domains.

Figure 2 (a) and (b) show mapped domains of function $f=\mathrm{Z}^{-1} \mathrm{C}_{1} \mathrm{C}_{2} \mathrm{C}_{3} \mathrm{C}_{4}$.

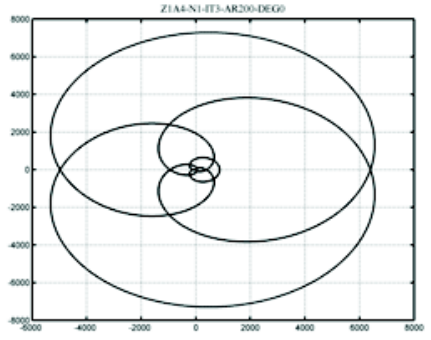

Figure 2 (a)

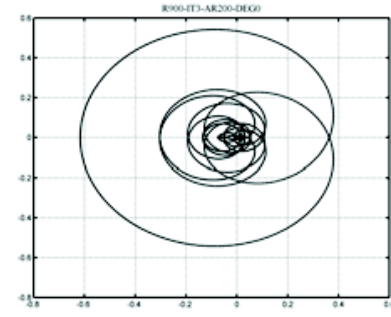

Figure 2 (b)

Figure 2 (a) shows the quasi-perodic orbit becoming chaotic orbit as shown in Figure 2 (B) when the parameters in parametric space change.

\section{Symmetry Broken and Chaos}

The parameters in the parametric space correlate to one another when an original domain becoming a chaotic domain. We define the transition from a stable to a chaotic domain based on the change of domain boundries by increasing the order of iteration. For example, when the iteration order changes from 10 to 1000 iterations and the low-iterated fractal bondaries of the original domain remain the same, we then claim that this is a stable domain. On the opposite, when the fractal boundaries change with iterantion order, then original domain becoming a chaotic domain with the given set of parameters. In the following, we will examie the impact of normalized energy level and normailzed phase factor on the symmetry broken of original domains and 
transition from stable domains to chaotic domains. The compound influence of these two parameters on the transitions is also illustrated.

\subsection{Normalized Energy Level (parameter: a)}

Figure 3 (a) through $(d)$ show the original domains of unit circle $(R=1)$ with normalized energy level $\mathrm{a}=0.01$ in Figure $3(\mathrm{a}), \mathrm{a}=0.1$ in Figure $3(\mathrm{~b}), \mathrm{a}=0.2$ in Figure 3 (c) and $\mathrm{a}=1$ Figure 3 (d).

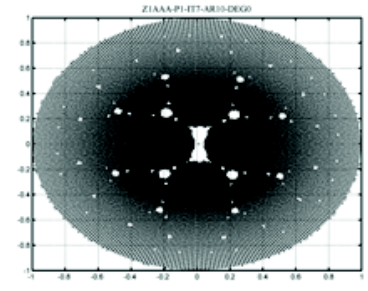

Figure 3 (a) $\mathrm{a}=0.01$

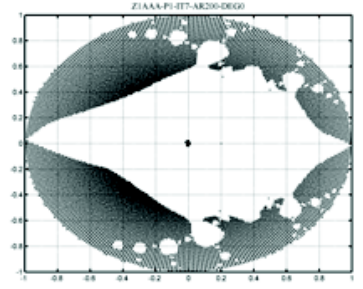

Figure 3 (c) $\mathrm{a}=0.2$

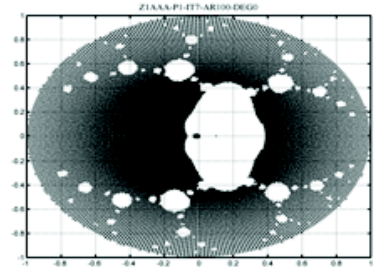

Figure $3(\mathrm{~b}) \mathrm{a}=0.1$

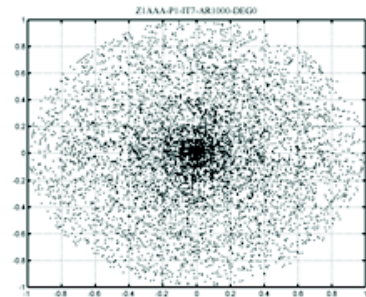

Figure $3(d) a=1$

We observe that the original domain showing symmetrical characteristics when the value of normalized energy level is small $(\mathrm{a}=0.01)$ as shown in Figure 3 (a). As the value of normalized energy level increases from 0.1 to 1 , the original domain becomes gradually to an asymmetrical fractal pattern and eventually become a chaotic pattern. This observation also applied to the unit circles of original domains at other functional orders.

\subsection{Normalized Phase factor ( parameter: $\exp \left(g_{i}(z)\right)$ )}

In the following, we examine $4^{\text {th }}$ order domains as shown in Figure 1 with parameter of normalized energy level $\mathrm{a}=0.1$ and normalized phase factor at 88 degrees when the original domain remains a stable domain and at 89 degrees when the original domain becomes a chaotic domain. These two specific degrees are defined as follows:

$$
\mathrm{g}(\mathrm{z})=\pi * \mathrm{i} *(88 / 180) \quad \text { and } \quad \mathrm{g}(\mathrm{z})=\pi * \mathrm{i} *(89 / 180)
$$

We examine the original domains based on the order of iterations as shown in Figures 4 (a) through (d) for the case of 88 degrees and in Figures 5 (a) through (f) for the case of 89 degrees. 


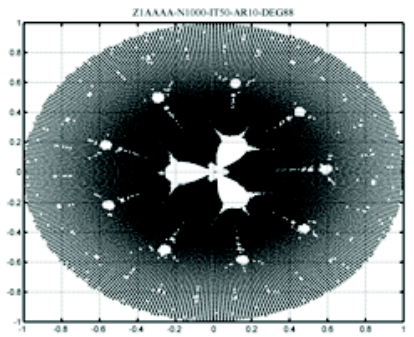

Figure 4 (a) iteration $=50$

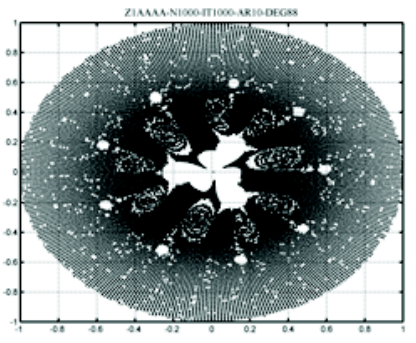

Figure $4(\mathrm{c})$ iteration $=1000$

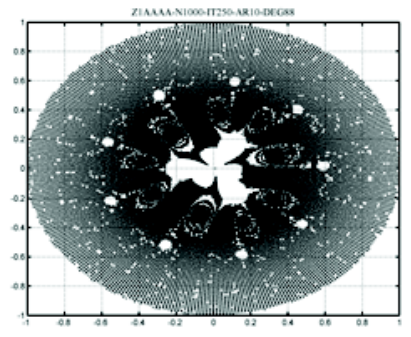

Figure $4(\mathrm{~b})$ iteration $=250$

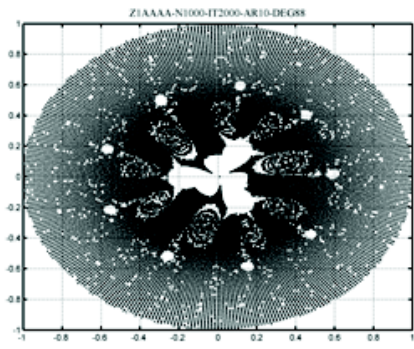

Figure $4(d)$ iteration $=2000$

Comparing with Figure 1 (c), Figure 4 (a) shows that the low-iterated portion of fractals at center of unit circle is rotated at 88 degrees while the bulbs extended from individual fractal branches lag an angle against the central portion of fractal pattern. This is another symmetry-broken mechanism by the normalized phase factor, $\exp (\mathrm{g}(\mathrm{z}))$. Also, a phenomenon similar to frequency-doubling is that the boundries of low-iterated portion of fractals at center is becoming a six-branched pattern instead of original 3-branched pattern. We call this phenomenon as branch-doubling. When the iternation number increases, we observe spirals formed to connect the extended bulbs back to the original branches as shown in Figure 4 (b) (iteranion number = 250). As the iternation numbers are further increased as Figure 4 (c) and Figure 4 (d), the central portion of fractal remains in the same fractal boundaries and only the turns of connected spiral increases and more extended bulbs around the inner boundary of the unit circle $(\mathrm{R}=1)$ appear. We claim that this set of parameters, namely, $\mathrm{a}=0.1$ and degree $=88$, defines a stable domain.

When the rotation angle increases from 88 degrees to 89 degrees, as shown in Figure 5, we observe that the branch-doubling as shown in Figure 5 (b) and Figure 5 (c) are not stopping at particular iteration, these newly growing branches further extend and connect to the angle-lagged exteneded bulbs. As the iteration number is equal to 100 shown in Figure 5 (d), we observe the spirals, as shown in Figure 4, appear and compete for connecting to extended bulbs. As the iteration number increases to 200, shown in Figure 5 (e), the 
branch-doubling and bulb-connecting competetion are further developed and eventually become a chaotic domain as shown in Figure 5 (f). This indicates that all points in the original domain $(1 \leqq \mathrm{R})$ will eventually diverge after a given number of iteration.

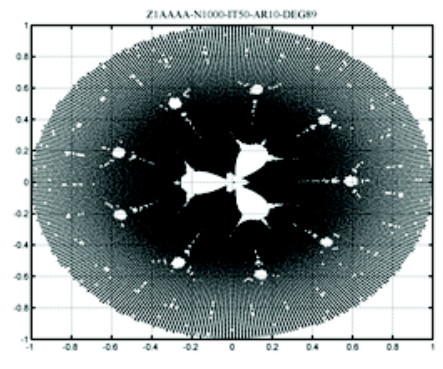

Figure $5(\mathrm{a})$ iteration $=50$

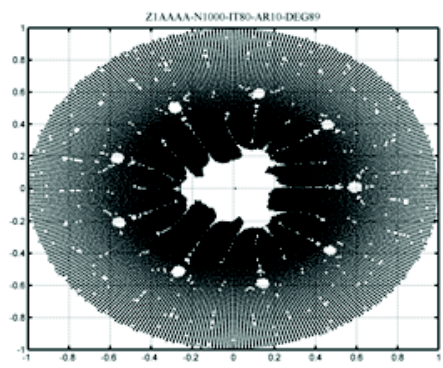

Figure 5 (c) iteration $=80$

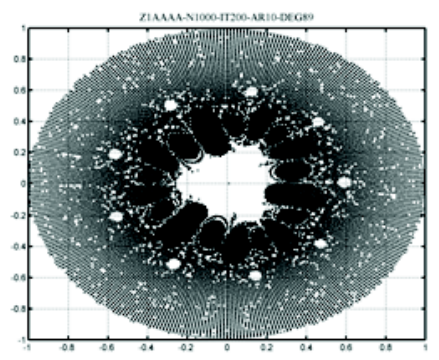

Figure 5 (e) iteration $=200$

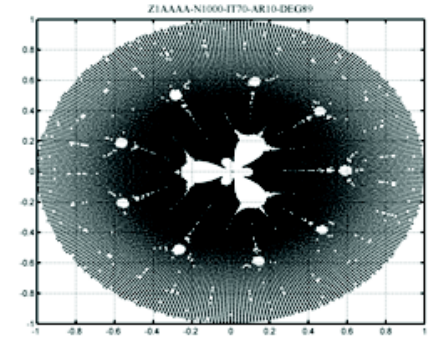

Figure $5(\mathrm{~b})$ iteration $=70$

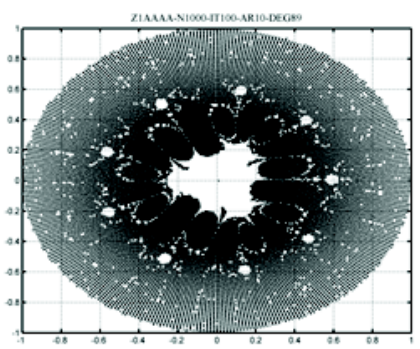

Figure $5(\mathrm{~d})$ iteration $=100$

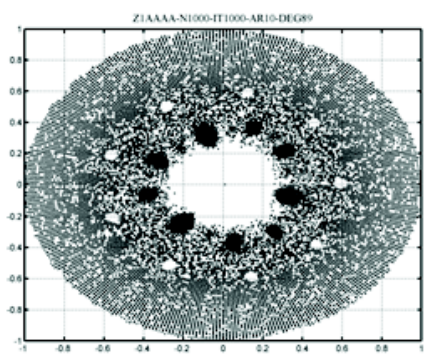

Figure $5(\mathrm{f})$ iteration $=1000$

As the normalized energy level increases, the chaotic transitions occur at lower rotation angles of the normalized phase factor, $\exp (\mathrm{g}(\mathrm{z}))$. Table 1 illustrates the cases for $\mathrm{a}=0.1, \mathrm{a}=0.2$, and $\mathrm{a}=0.3$. As normalized energy level increases to a crital value, the origianal domains may become chaotic domains without any rotation applied. 
Table 1 Chaotic Transition in the parametric space

\begin{tabular}{|c|c|c|c|}
\hline Parameters $(\mathrm{a}, \mathrm{g}(\mathrm{z}))$ & $\mathrm{a}=0.1$ & $\mathrm{a}=0.2$ & $\mathrm{a}=0.3$ \\
\hline Transition Angle & 73 degrees & 52 degrees & 32 degrees \\
\hline
\end{tabular}

\section{Conclusions}

In this paper we examined the transiton to chaotic status of the original domains of function $f=\mathrm{h}(\mathrm{z}) \prod_{\mathrm{i}}\left\{\exp \left(\mathrm{g}_{\mathrm{i}}(\mathrm{z})\right)\left[\left(\mathrm{a}_{\mathrm{i}} \mathrm{z}\right) /\left(1-\overline{\mathrm{a}}_{\mathrm{i}} \mathrm{z}\right)\right]\right\}$. The normalized energy level and normalized phase factor are two paramters in the parametric space of this function induce symmtry broken in the fractal patterns. Acompanying with symmetry broken, we observed the trasitions from to chaotic domains under the influnced of combined effect of these two parameters. Brach-doubling and bulb-connecting competion are the observed phenomena during the transition. We will further investigate mathematical and physical implications of these computational observations.

\section{References}

[1] Benoit B. Mandelbrot, The Fractal Geometry of Nature, New York, W.H. Freeman and Company, 1983.

[2] K. Falconer, Fractal Geometry: Mathematical Foundations and Applications, New York, John Wiley \& Sons, 1990.

[3] J. Milnor, Dynamics in One Complex Variable, Vieweg, 2000.

[4] A. Fathi and J. -C. Yoccoz (Eds.), Dynamical Systems: Michael Herman Memorial Volume, Cambridge University Press, Feb. 2006.

[5] David C. Ni and C. H. Chin, " $\mathrm{Z}^{-1} \mathrm{C}_{1} \mathrm{C}_{2} \mathrm{C}_{3} \mathrm{C}_{4}$ System and Application", TIENCS workshop, Singapore, August 1-5, 2006.

[6] David C. L. Ni and C. H. Chin, "A Novel Approach for Designing Fractal Antennas", Proc. of International Conference on Wireless Information Networks and Systems, pp. 157-160, (Eds. S.O. Mohammand et al.), Barcelona, Spain, July 2007.

[7] David C. Ni and C. H. Chin, "Broadband Fractal Antennas", Proc. of International Symposium on Antennas and Propagation, Taipei, Taiwan, pp. 68 (Abstract) 27-30 October 2008.

[8] C. H. Chin, "Hypertranscendental Geometry”, Preprint NCTU-IMS-0801, Taiwan, R.O.C.

[9] David C. Ni and C. H. Chin, "Herman Ring Classification on Function $\mathrm{h}(\mathrm{z}) \prod_{\mathrm{i}}\left\{\exp \left(\mathrm{g}_{\mathrm{i}}(\mathrm{z})\right)\left[\left(\mathrm{a}_{\mathrm{i}}-\mathrm{z}\right) /\left(1-\overline{\mathrm{a}}_{\mathrm{i}} \mathrm{z}\right)\right]\right\}$ ", accepted for publication in the 5 th International Conference 2009 - Dynamical Systems and Applications, Constantza, Romania, June 2009 